\title{
Cardiopatías congénitas, resultados del manejo perioperatorio en 18 meses. Experiencia en el Departamento de Cardiocirugía Centro Materno Infantil. UNA
}

\author{
CARÍSIMO M., SZWAKO R., GARAY N., WILDO PINO, GAONA N., EGUSQUIZA P., \\ JAROLÍN J, GONZÁLEZ ÁVILA F, SCIACCA R, PANIZZA C, MONTANER M. ${ }^{1}$ \\ 1. Departamento de Cardiocirugía Infantil. Cátedra y Servicio de Pediatría. Centro Materno Infantil. Facultad de Ciencias \\ Médicas. Universidad Nacional de Asunción.
}

\begin{abstract}
Congenital heart defects, perioperative manegement results in 18 months. Experience of the Maternal and Child Center Cardiosurgery Section
\end{abstract}

Introduction: Survival of patients with congenital heart defects has improved in recent years thanks to improved diagnostic, surgical, anesthetic, and extracorporeal circulation techniques, and the arrival of new and more selective cardioactive drugs. Objectives: To describe perioperative care and postoperative progress of patients following surgery performed in the department of cardiac surgery and pediatrics department of the Centro Materno Infantil (CMI) of the National University's School of Medical Sciences over a period of 18 months, and its relationship to the type of surgery performed (elective or emergency) and the patient's presurgical physical status classification of the American Society of Anesthesiologists (ASA-PS). Materials and Methods: A retrospective, descriptive, observational study with an analytical component using the medical records of patients operated on in the department of cardiac surgery between January 2007 and June 2008. Results: Of the 91 patients undergoing surgery, 38 (42\%) were male, and 53 (58\%) were female, with ages ranging from 2 days to 18 years. Uncomplicated heart disease was found in 54 patients (59.3\%), and complications confirmed by color Doppler echocardiography were found in 37 (40.7\%). Cardiac catheters were needed before surgery by 3 patients (3.3\%). All surgeries (100\%) were performed under balanced general anesthesia. Of the 91 patients operated on, 42 (46.2\%) received extracorporeal circulation (ECC) and 49 (53.8\%) did not. Of the 42 patients who received ECC, all involved corrective surgery, with 40 (96.2\%) of those being elective, and 2 (4.8\%) emergency surgery, while 3 (7.1\%) were reoperated and 2 (4.8\%) died. Complications appeared in 16 patients (38.1\%), with 39 (92.9\%) requiring mechanically assisted ventilation (MAV) for less than 24 hours, and 3 (7.1\%) who received MAV for more than 24 hours. Aortic clamping time ranged from 13 to 167 minutes (mean $49.1 \mathrm{~min}$ ) and ECC times from 20 to 253 minutes (mean $71.9 \mathrm{~min}$ ). Plasma ultrafiltration (pUF) was done in all (100\%) of patients operated on who received ECC, all of whom also received prophylactic aprotinin for bleeding. Of the 49 patients operated on without ECC, 30 (61.2\%) were corrective surgeries, 19 (38.8\%) were palliative, 31 (63.3\%) were elective, 18 (36.7\%) were emergency surgery, 1 (2.1\%) was a reoperation,

Correspondencia a:

Dr. Wildo Pino

E-mail: wildopino@hotmail.com 
and 10 (16.3\%) died. Complications appeared in 18 patients (36.7\%), with 39 (79.6\%) requiring MAV for less than 24 hours, and 10 (20.4\%) receiving MAV for more than 24 hours. Of the patients operated on, 34 developed complications, 28\% in elective surgeries and 65\% in emergency surgeries; by type these were 50\% were hemodynamic; 35.3\% respiratory, 23.5\% neurological, 14.7\% infectious, 14.7\% metabolic, 11.8\% hematological, and $11.8 \%$ renal. Conclusions: Morbidity and mortality were directly related to two factors: being emergency surgery, and the presurgical physical status classification of the patient as per the ASA-PS. There were no deaths in the elective surgeries, and the patients who died had an ASA 4 preoperative physical status classification. Intraoperative use of pUF and aprotinin contributed to the favorable progress of the patients operated on with use of ECC.

(Key words: Congenital heart defects, perioperative care, extracorporeal circulation, cardiac surgery, child). Pediatr. (Asunción), Vol. 36 Nº 3; 2009

\section{RESUMEN}

Introducción: La sobrevida de los pacientes con cardiopatías congénitas ha mejorado en los últimos años gracias al perfeccionamiento en las técnicas diagnósticas, quirúrgicas, anestésicas y de perfusión, así como al advenimiento de nuevas y más selectivas drogas cardiológicas. Objetivos: Describir el manejo perioperatorio y la evolución postoperatoria de las cirugías realizadas en el Departamento de Cardiocirugía de la Cátedra y Servicio de Pediatría del Centro Materno Infantil (CMI), FCM-UNA en 18 meses de atención y su relación con el carácter de la cirugía (electiva o de urgencia), y el estado físico preoperatorio del paciente, definido por los criterios de la American Society of Anesthesiologists (ASA). Materiales y Métodos: Estudio observacional, retrospectivo, descriptivo, con componente analítico; se utilizaron fichas clínicas de pacientes operados en el Departamento de Cardiocirugía de enero de 2007 a junio de 2008. Resultados: Fueron operados 91 pacientes, 38 de sexo masculino (42\%) y 53 de sexo femenino (58\%), con edades comprendidas entre 2 días y 18 años. Las cardiopatías fueron 54/91 (59,3\%) patologías simples y 37/91 (40,7\%) patologías complejas. Todas con confirmación diagnóstica preoperatoria por ecocardiografía Doppler color. Precisaron cateterismo cardíaco preoperatorio 3/91 pacientes (3,3\%). El 100\% de las cirugías se realizaron con anestesia general balanceada. De los 91 pacientes operados, 42 (46,2\%) fueron sometidos a circulación extracorpórea (CEC) y 49 (53,8\%) sin CEC. De los 42 pacientes operados con CEC, todas fueron cirugías correctivas, 40 (95,2\%) electivas, 2 (4,8\%) de urgencia, 3 (7,1\%) reintervenciones y 2 (4,8\%) fallecieron. Presentaron complicaciones 16 pacientes (38,1\%), 39 (92,9\%) requirieron Asistencia Respiratoria Mecánica (ARM) menos de 24 horas y 3 (7,1\%) más de 24 horas. Los tiempos de clampado aórtico variaron de 13 a 167 minutos (media 49,1) y los tiempos de CEC de 20 a 253 minutos (media 71,9). Se realizó ultrafiltrado plasmático (UFP) en el 100\% de los pacientes operados con CEC y todos ellos recibieron aprotinina para profilaxis del sangrado. De los 49 pacientes operados sin CEC, 30 (61,2\%) fueron cirugías correctivas, 19 (38,8\%) paliativas, 31 (63,3\%) electivas, 18 (36,7\%) urgencias, 1 (2,1\%) reintervención y 10 (16,3\%) fallecieron. Presentaron complicaciones 18 pacientes (36,7\%), 39 (79,6\%) precisaron ARM menos de 24 horas y 10 (20,4\%) más de 24 horas. Del total de pacientes operados, 34 presentaron alguna complicación, el 50\% fue de tipo hemodinámico, 35,3\% respiratorio, 23,5\% neurológico, 14,7\% infeccioso, 14,7\% metabólico, 11,8\% hematológico y 11,8\% renal. El 28\% de las complicaciones se presentaron en las cirugías electivas y el 65\% en las urgencias. Conclusiones: La morbimortalidad de los pacientes estuvo directamente relacionada con dos factores: el carácter urgente de la cirugía y el estado físico preoperatorio del paciente (evaluado según criterio de la American Society of Anesthesiologists, ASA). No hubo mortalidad en las cirugías electivas y los pacientes fallecidos correspondieron a un estado físico preoperatorio ASA 4. La utilización intraoperatoria de UFP y aprotinina contribuyó para la buena evolución de las cirugías con CEC.

(Palabras clave: Cardiopatías congénitas, atención perioperativa, circulación extracorpórea, cirugía torácica, niño).

Pediatr. (Asunción), Vol. 36 No 3; 2009

ESTE TRABAJO LO PUEDE ENCONTRAR EN EXTENSO EN WWW.SciELO.ORG 